\title{
COUNTEREXAMPLE TO THE SPECTRAL MAPPING THEOREM FOR THE EXPONENTIAL FUNCTION ${ }^{1}$
}

\author{
J. HEJTMANEK AND HANS G. KAPER
}

\begin{abstract}
An example is given of an unbounded operator in a Hilbert space which generates a strongly continuous semigroup and for which the spectral mapping theorem for the exponential function does not hold. The spectra of both the generator and the semigroup are determined explicitly.
\end{abstract}

1. Introduction. Let $X$ be a Banach space and $W=[W(t): t \geqslant 0]$ a strongly continuous semigroup of bounded linear operators on $X$ with generator $A, W(t)=$ $\exp (A t)$. The spectral bound of the generator, $s$, is

$$
s=\sup \{\operatorname{Re} \lambda: \lambda \in \sigma(A)\}
$$

the type of the semigroup, $\omega_{0}$, is

$$
\omega_{0}=\inf \{\omega \in \mathbf{R}: \exists M \geqslant 1, \forall t \geqslant 0,\|W(t)\| \leqslant M \exp (\omega t)\} .
$$

It is well known that $s \leqslant \omega_{0}$. Furthermore, $r(W(t))=\exp \left(\omega_{0} t\right)$, where $r$ is the spectral radius.

In general, $\exp (\sigma(A) t) \subset \sigma(W(t)) \backslash\{0\}$. The identity

$$
\exp (\sigma(A) t)=\sigma(W(t)) \backslash\{0\} \quad \text { for all } t \geqslant 0,
$$

is known as the Spectral Mapping Theorem (SMT) for the exponential function. SMT is true if $\left[W(t): t \geqslant t_{0}\right]$ is uniformly continuous for some $t_{0}>0$ (see Davies [1980, Theorem 2.19]). SMT cannot be true if $s<\omega_{0}$.

Semigroups for which $s<\omega_{0}$ have been given by Hille and Phillips [1957, XXIII, 16], Foias [1973], Zabczyk [1975], Greiner, Voigt and Wolff [1981], Wolff [1981], Nagel [1982], and Hoppen [1985]. In each case, tine spectrum. of the generator could be computed, but not the spectrum of the semigroup; the type of the semigroup was computed from the definition. In this article we present an example of a semigroup with $s<\omega_{0}$, for which the spectra of both the generator and the semigroup can be computed. The example incorporates aspects of the examples of Foias and Zabczyk.

This research was motivated by our work in linear transport theory, where SMT plays an important role in the determination of the asymptotic behavior of particle distribution functions; see Kaper, Lekkerkerker and Hejtmanek [1982, Chapters 12 and 13], Greiner [1982], Voigt [1984], and Kaper and Hejtmanek [1984]. General

Received by the editors September 19, 1984 and, in revised form, February 25, 1985.

1980 Mathematics Subject Classification. Primary 47D05.

${ }^{1}$ This work was supported in part by the Applied Mathematical Sciences subprogram of the Office of Energy Research, U. S. Department of Energy, under contract W-31-109-Eng-38. 
references for the theory of linear semigroups are Hille and Phillips [1957], Davies [1980], Goldstein [1983], and Pazy [1983]; see also Dunford and Schwartz [1958, §VIII.1], Kato [1966, Chapter X], and Reed and Simon [1975, §X.8]. We shall make use of an SMT-related result of Gearhart [1978]; see also Herbst [1983], Howland [1984], and Pruess [1984].

2. Definitions and basic properties. Let $\left\{H_{k}: k=2,3, \ldots\right\}$ be a sequence of Hilbert spaces and $H=\oplus_{k=2}^{\infty} H_{k}$. Let $B_{k} \in L\left(H_{k}\right)$ be such that $\left\|B_{k}\right\|=1$ and $\sigma\left(B_{k}\right)=\{0\}$. We define $A_{k} \in L\left(H_{k}\right)$ by the expression

$$
A_{k}=2 \pi i k \dot{I}_{k}+B_{k},
$$

where $I_{k}$ is the identity in $L\left(H_{k}\right)$, and we define the unbounded operator $A$ on $\operatorname{dom} A \subset H$ by the direct sum

$$
A=\bigoplus_{k=2}^{\infty} A_{k} .
$$

The operator $A$ is closed and $\operatorname{dom} A=\left\{x \in H: \sum_{k=2}^{\infty} k^{2}\left\|x_{k}\right\|^{2}<\infty\right\}$. The resolvent of $A$, which is given by

$$
R(\lambda, A)=\bigoplus_{k=2}^{\infty} R\left(\lambda, A_{k}\right), \quad \lambda \in \rho(A),
$$

satisfies the norm identity

$$
\|R(\lambda, A)\|=\sup \left\{\left\|R\left(\lambda, A_{k}\right)\right\|: k=2,3, \ldots\right\} .
$$

The spectrum of $A$ is easily computed. Because $2 \pi i k \in \sigma\left(A_{k}\right)$ for each $k=2,3, \ldots$, we have $2 \pi i k \in \sigma(A)$ for $k=2,3, \ldots$. For any point $\lambda \neq 2 \pi i k, k=2,3, \ldots$, we have

$$
R\left(\lambda, A_{k}\right)=(\lambda-2 \pi i k)^{-1}\left(I_{k}-(\lambda-2 \pi i k)^{-1} B_{k}\right)^{-1} .
$$

Because $\left\|B_{k}\right\|=1$ for all values of $k$, and $|\lambda-2 \pi i k| \geqslant 1+\varepsilon, \varepsilon>0$, for all but at most one value $k_{0}$ of $k$, where $\lambda-2 \pi i k_{0} \in \rho\left(B_{k_{0}}\right)$, it follows that $\sup \left\{\left\|R\left(\lambda, A_{k}\right)\right\|\right.$ : $k=2,3, \ldots\}<\infty$ and, therefore, $\lambda \in \rho(A)$. Hence,

$$
\sigma(A)=\{2 \pi i k: k=2,3, \ldots\},
$$

and $s=0$.

If $\lambda \in \mathbf{C}$ and $|\lambda-2 \pi i k|>1$, then $\left\|R\left(\lambda, A_{k}\right)\right\|<(|\lambda-2 \pi i k|-1)^{-1}$. If, in addition, $\operatorname{Re} \lambda>1$, then $\left\|R\left(\lambda, A_{k}\right)\right\| \leqslant(\operatorname{Re} \lambda-1)^{-1}$ for $k=2,3, \ldots$ Therefore,

$$
\|R(\lambda, A)\| \leqslant(\operatorname{Re} \lambda-1)^{-1}, \quad \lambda \in \mathrm{C}, \operatorname{Re} \lambda>1 .
$$

It follows from the Hille-Yosida Theorem that $A$ is the generator of a semigroup $W=[W(t): t \geqslant 0]$, with $W(t)=\exp (A t)$ given by

$$
W(t)=\bigoplus_{k=2}^{\infty} W_{k}(t), \quad t \geqslant 0,
$$

where

$$
W_{k}(t)=\exp (2 \pi i k t) \exp \left(B_{k} t\right) .
$$

Clearly, $\|W(t)\| \leqslant e^{t}$ for $t \geqslant 0$, and $\omega_{0} \leqslant 1$. 
For special choices of $H_{k}$ and $B_{k}$, Zabczyk showed that $\|W(t)\|=e^{t}$ for $t \geqslant 0$. Foias, choosing different $H_{k}$ and $B_{k}$, showed that $e^{\alpha t} \in \sigma(W(t))$ for all $\alpha \in(0,1)$. (Here we have changed the definitions to get agreement with Zabczyk's.) In both cases, therefore, $\omega_{0}=1$.

We take the same choices of $H_{k}$ and $B_{k}$ as Zabczyk:

$$
H_{k}=\mathbf{C}^{k}, \quad B_{k}=\operatorname{mat}\left[b_{k: i, j}: i, j=1, \ldots, k\right],
$$

where $b_{k ; i, i+1}=1$ for $i=1, \ldots, k-1, b_{k ; i, j}=0$ otherwise. One verifies that $\left\|B_{k}\right\|$ $=1, \sigma\left(B_{k}\right)=\{0\}$, and $B_{k}^{k}=0$. The resolvent of $B_{k}$ is given by a finite sum

$$
R\left(\lambda, B_{k}\right)=\sum_{n=0}^{k-1} \lambda^{-(n+1)} B_{k}^{n}, \quad \lambda \in \mathbf{C}, \lambda \neq 0 .
$$

3. Bounds for the resolvent. Our aim is to apply the following theorem of Gearhart.

TheOREM 1. Let $W=[W(t): t \geqslant 0]$ be a semigroup with generator $A$ in a Hilbert space. Then $e^{z} \in \rho(W(1))$ iff

(i) $z+2 \pi i l \in \rho(A)$ for all $l \in \mathbf{Z}$, and

(ii) $\sup \{\|R(z+2 \pi i l, A)\|: l \in \mathbf{Z}\}<\infty$.

Proof. See Gearhart [1978], Herbst [1983], Howland [1984], and Pruess [1984].

Condition (i) is satisfied for any point $z \in \mathbf{C}$ that is not an integer multiple of $2 \pi i$. Condition (ii) requires estimates of the resolvent along lines parallel to the imaginary axis. It follows from (2.4) and the definition of $A_{k}$ that

$$
\|R(z+2 \pi i l, A)\|=\sup \left\{\left\|R\left(z+2 \pi i(l-k), B_{k}\right)\right\|: k=2,3, \ldots\right\},
$$

whenever $z+2 \pi i l \in \rho(A)$.

Lemma 2. For all $\lambda \in \mathrm{C}$ with $|\lambda|>0$ and for all $k=2,3, \ldots$, we have the inequalities

$$
g(|\lambda|, k) \leqslant\left\|R\left(\lambda, B_{k}\right)\right\| \leqslant h(|\lambda|, k),
$$

where

$$
g(x, k)=\left(\sum_{j=1}^{k} x^{-2 j}\right)^{1 / 2}, \quad h(x, k)=\sum_{j=1}^{k} x^{-j}
$$

for $x>0$ and $k=2,3, \ldots$.

Proof. The upper bound in (3.2) is trivial. Let $e_{k}=(0, \ldots, 0,1)^{T}$. The vectors $B_{k}^{n} e_{k}, n=0, \ldots, k-1$, are orthonormal in $H_{k}$, so

$$
\left\|R\left(\lambda, B_{k}\right)\right\| \geqslant\left\|R\left(\lambda, B_{k}\right) e_{k}\right\| \geqslant\left(\sum_{j=1}^{k}|\lambda|^{-2 j}\right)^{1 / 2} .
$$


The functions $g$ and $h$ have the following properties: (i) for $x$ fixed, $g$ and $h$ increase as functions of $k$; (ii) for $k$ fixed, $g$ and $h$ decrease as functions of $x$; (iii) $g(1, k)=\sqrt{k}, h(1, k)=k ;$ and (iv) for $x>1, \lim _{k \rightarrow \infty} g(x, k)=\left(x^{2}-1\right)^{-1 / 2}$, $\lim _{k \rightarrow \infty} h(x, k)=(x-1)^{-1}$. These properties imply that, for fixed $k$,

$$
\begin{gathered}
\left\|R\left(\lambda, B_{k}\right)\right\|=|\lambda|^{-1}\left(1+O\left(|\lambda|^{-1}\right)\right) \quad \text { as }|\lambda| \rightarrow \infty, \\
\left\|R\left(\lambda, B_{k}\right)\right\|=|\lambda|^{-k}(1+O(|\lambda|)) \text { as }|\lambda| \rightarrow 0 .
\end{gathered}
$$

Furthermore, for $k=2,3, \ldots$,

$$
\begin{gathered}
\left\|R\left(\lambda, B_{k}\right)\right\| \geqslant \sqrt{k} \quad \text { for all }|\lambda| \leqslant 1, \\
\left\|R\left(\lambda, B_{k}\right)\right\| \leqslant(\rho-1)^{-1} \text { for all }|\lambda| \geqslant \rho>1 .
\end{gathered}
$$

In the next section we apply these estimates to calculate the supremum in (3.1).

4. Spectrum of the semigroup. Let $S=\bigcup_{m \in \mathbf{Z}}(D+2 \pi i m)$, where $D$ is the punctured unit disc, $D=\{z \in \mathbf{C}: 0<|z| \leqslant 1\}$. Clearly, $S \subset \rho(A)$. If $z \in S$, then there exists, for every $k \in\{2,3, \ldots\}$, an $l \in \mathbf{Z}$ such that $|z+2 \pi i(l-k)| \leqslant 1$. Therefore, using (3.5), we see that

$$
\sup \left\{\left\|R\left(z+2 \pi i(l-k), B_{k}\right)\right\|: l \in \mathbf{Z}, k=2,3, \ldots\right\}=\infty,
$$

and Theorem 1 yields $e^{z} \in \sigma(W(1))$.

If $z \in \mathbf{C} \backslash \bar{S}$, then $|z+2 \pi i(l-k)| \geqslant \operatorname{dist}(z,\{2 \pi i m: m \in \mathbf{Z}\})=\rho>1$ for all $k=2,3, \ldots$ and $l \in \mathbf{Z}$. Therefore, using (3.6), we see that

$$
\sup \left\{\left\|R\left(z+2 \pi i(l-k), B_{k}\right)\right\|: l \in \mathbf{Z}, k=2,3, \ldots\right\}<\infty,
$$

and Theorem 1 yields $e^{z} \in \rho(W(1))$.

Combining these results, we find

$$
\sigma(W(1)) \backslash\{0\}=e^{\sigma(A)} \cup\left\{e^{z}: 0<|z| \leqslant 1\right\} .
$$

Here, $e^{\sigma(A)}$ consists of the single point $\left\{e^{z}: z=0\right\}$.

Let $t>0$. Then Theorem 1 implies that $e^{t z} \in \rho(W(t))$ iff (i) $z+(2 \pi / t) i l \in \rho(A)$ for all $l \in \mathbf{Z}$, and (ii) $\sup \{\|R(z+(2 \pi / t) i l, A)\|: l \in \mathbf{Z}\}<\infty$. Here,

$$
\|R(z+(2 \pi / t) i l, A)\|=\sup \left\{\left\|R\left(z+2 \pi i(l / t-k), B_{k}\right)\right\|: k=2,3, \ldots\right\} .
$$

We distinguish two cases: $t$ rational and $t$ irrational.

Let $t$ be rational, $t=p / q$. Then

$$
\sigma(W(p / q))=(\sigma(W(1 / q)))^{p} .
$$

If $z \in S$ and $|z| \leqslant 1$, then there exists, for every $k \in\{q, 2 q, \ldots\}$, an $l \in \mathbf{Z}$ such that $|z+2 \pi i(q l-k)| \leqslant 1$. Therefore,

$$
\sup \left\{\left\|R\left(z+2 \pi i(q l-k), B_{k}\right)\right\|: l \in \mathbf{Z}, k=2,3, \ldots\right\}=\infty,
$$

and $e^{t z} \in \sigma(W(t))$. A similar procedure yields $e^{t z} \in \sigma(W(t))$ if $z \in S$ and $\mid z+$ $2 \pi i m \mid \leqslant 1$ for some $m \in \mathbf{Z}$. 
If $z \in \mathbf{C} \backslash \bar{S}$, then $|z+2 \pi i(q l-k)| \geqslant \operatorname{dist}(z,\{2 \pi i m: m \in \mathbf{Z}\})=\rho>1$ for all $k=2,3, \ldots$ and $l \in \mathbf{Z}$. Therefore,

$$
\sup \left\{\left\|R\left(z+2 \pi i(q l-k), B_{k}\right)\right\|: l \in \mathbf{Z}, k=2,3, \ldots\right\}<\infty,
$$

and $e^{t z} \in \rho(W(t))$.

Summarizing, we have

$$
\sigma(W(t)) \backslash\{0\}=e^{\sigma(A) t} \cup\left\{e^{z t}: 0<|z| \leqslant 1\right\},
$$

whenever $t$ is rational. If $t=p / q$, where $p$ and $q$ are relatively prime, then $e^{\sigma(A) t}$ is the finite set $\left\{e^{z t}: z=2 \pi i k, k=0, \ldots, q-1\right\}$ of $q$ points on the unit circle.

Let $t$ be irrational. The sequence $\{l / t: l=1,2,3, \ldots\}$ is uniformly distributed modulo 1; cf. Cassels [1957, IV]. Hence, for every $z=\alpha+2 \pi i \beta$ with $0<|\alpha|<1$ and $0 \leqslant \beta \leqslant 1$, there exist infinitely many $k \in\{2,3, \ldots\}$ and $l \in \mathbf{Z}$ such that

$$
|l / t-k+\beta| \leqslant\left(1-\alpha^{2}\right)^{1 / 2} .
$$

Each such pair $(k, l)$ generates a point $z+2 \pi i(l / t-k)$ that belongs to $D$ and therefore to $S$, so $e^{t z} \in \sigma(W(t))$. Furthermore, $e^{\sigma(A) t}$ is a dense set on the unit circle. Because $\sigma(W(t))$ is closed, it follows that

$$
\sigma(W(t)) \backslash\{0\}=\left\{e^{t z}:|\operatorname{Re} z| \leqslant 1,0 \leqslant \operatorname{Im} z \leqslant 2 \pi\right\},
$$

whenever $t$ is irrational.

Thus, $\sigma(W(t))$ is known for all $t>0$.

5. Remarks. Because $-A$ also generates a semigroup, $A$ is the generator of a group, and

$$
\|R(\lambda, A)\| \leqslant(|\operatorname{Re} \lambda|-1)^{-1}, \quad \lambda \in \mathbf{C},|\operatorname{Re} \lambda|>1 .
$$

Furthermore,

$$
\|R(\lambda, A)\| \leqslant|\operatorname{Im} \lambda|^{-1}, \quad \lambda \in \mathbf{C}, \operatorname{Im} \lambda<0 .
$$

Hence, $i A$ also generates a semigroup.

The graph of $\|R(\lambda, A)\|, \lambda \in \rho(A)$, has the following qualitative behavior. As $|\operatorname{Re} \lambda| \rightarrow \infty$, it decreases to zero like $|\operatorname{Re} \lambda|^{-1}$; as $\operatorname{Im} \lambda \rightarrow-\infty$, it decreases to zero like $|\operatorname{Im} \lambda|^{-1}$. It is bounded in the strip $\{\lambda \in \mathbf{C}:|\operatorname{Re} \lambda| \leqslant 1, \operatorname{Im} \lambda \geqslant 0\}$, except in the discs $D+2 \pi i k, k=2,3, \ldots$, where $\|R(z, A)\|=|z-2 \pi i k|^{-k}(1+O(|z-2 \pi i k|))$ as $z \rightarrow 2 \pi i k$.

If $A$ is rotated over an angle $\phi, 0<\phi<\pi / 2$, then we obtain a new operator $e^{i \phi} A$ for which SMT holds. This result is verified most easily from Theorem 1 if one uses the above qualitative results about the graph of $\|R(\lambda, A)\|$.

\section{REFERENCES}

J. W. S. Cassels [1957], An introduction to diophantine approximation, Cambridge Tracts in Math. and Math. Phys., no 45, Cambridge Univ. Press, New York.

E. B. Davies [1980], One-parameter semigroups, London Math. Soc. Monographs, vol. 15, Academic Press, New York.

N. Dunford and J. T. Schwartz [1958], Linear operators, Interscience, New York.

C. Foias [1973], Sur une question de M. Reghis, An. Univ. Timisoara 11, fasc. 2. 
L. Gearhart [1978], Spectral theory for contraction semigroups on Hilbert spaces, Trans. Amer. Math. Soc. 236, 385-394.

J. Goldstein [1983], Semigroups of linear operators and applications, Tulane University.

G. Greiner [1982], Asymptotics in linear transport theory, Semesterberichte Funktionalanalysis Tübingen, Sommersemester 1982, pp. 71-98.

G. Greiner, J. Voigt and M. Wolff [1981], On the spectral bound of the generator of semigroups of positive operators, J. Operator Theory 5, 245-256.

I. Herbst [1983], The spectrum of Hilbert space semigroups, J. Operator Theory 10, 87-94.

E. Hille and R. S. Phillips [1957], Functional analysis and semigroups, Colloq. Publ., vol. 21., Amer. Math. Soc., Providence, R.I.

N. Hoppen [1985], Examples of semigroups with $s<\omega_{0}$ in $L^{1}$-Banach spaces, Thesis, Institut für Mathematik, Universität Wien.

J. S. Howland [1984], On a theorem of Gearhart, Integral Equations Operator Theory 7, 138-142.

H. G. Kaper and J. Hejtmanek [1984], Recent advances on the reactor problem, (preprint).

H. G. Kaper, C. G. Lekkerkerker and J. Hejtmanek [1982], Spectral methods in linear transport theory, 0T5, Birkhäuser-Verlag, Basel.

T. Kato [1966], Perturbation theory for linear operators, Springer-Verlag, New York.

R. Nagel [1982], Zur Charakterisierung stabiler Operatorenhalbgruppen, Semesterberichte Funktionalanalysis Tübingen, Sommersemester 1982, pp. 99-120.

A. Pazy [1983], Semigroups of linear operators and applications to partial differential equations, SpringerVerlag, New York.

J. Pruess [1984], On the spectrum of $C_{0}$-semigroups, Trans. Amer. Math. Soc. 284, 847-857.

M. Reed and B. Simon [1975], Methods of modern mathematical physics, Vol. 2, Academic Press, New York.

M. Wolff [1981], A remark on the spectral bound of the generator of a semigroup of positive operators with applications in stability theory, Functional Analysis and Applications, Birkhäuser-Verlag, Basel, pp. 39-50.

J. Voigt [1984], Positivity in time dependent linear transport theory, Preprint, Fach. Mathematik, Universität Trier.

J. Zabczyk [1975], A note on $C_{0}$-semigroups, Bull. Acad. Polon. Sci. 23, 895-898.

Mathematics and Computer Science Division, Argonne National laboratory, Argonne, ILLINOIS 60439 (Current address of H. G. Kaper)

Current address (J. Hejtmanek): Mathematisches Institut der Universität Wien, Strudlhofgasse 4, A-1090 Wien, Austria 